\title{
Imaging in a Rare Case of Unilateral External and Middle Ear Anomalies Associated with Inner Ear Malformation
}

\author{
Anagha R. Joshi ${ }^{1} \quad$ Anisha O. Talreja ${ }^{1} \quad$ Mrinalini M. Rahatgaonkar ${ }^{1} \quad$ Harshal A. Badgujar $^{1}$ \\ 1Department of Radiology, Lokmanya Tilak Municipal General \\ Hospital and Medical College, Sion, Mumbai, Maharashtra, India \\ Address for correspondence Anisha O. Talreja, Department of \\ Radiology, Lokmanya Tilak Municipal General Hospital and Medical \\ College, Sion, Mumbai, Maharashtra, India \\ (e-mail: anishatalreja10@gmail.com).
}
Abstract
Keywords
- CT
- EAC atresia
- external
- middle ear
- inner ear malformations
$-M R I$

External ear malformations are commonly associated with middle ear malformations due to their closely related embryogenesis. Inner ear anomalies are less consistently seen due to their independent development. Cross-sectional imaging has come to play a critical role in the workup of congenital deafness in the pediatric age group. To optimize speech and language development, early diagnosis and treatment are very crucial. Identification of these anomalies is additionally important when operative intervention is being planned because they may complicate or perhaps preclude the surgery.

\section{Introduction}

One of the key causes of childhood disability worldwide is congenital deafness. External ear anomalies are often related to middle ear anomalies and infrequently with the inner ear. Imaging plays a vital role in its characterization and preoperative workup. Magnetic resonance imaging (MRI) and high-resolution computed tomography (HRCT) are complementary. CT helps in providing minute anatomical details, whereas MRI provides excellent delineation of the membranous labyrinth, facial and vestibulocochlear nerves, and brain. In this article, we have presented an exceedingly rare case of unilateral external and middle ear anomalies associated with labyrinthine anomaly and discussed how crucial the role of imaging is in diagnosing related anomalies before surgical decision-making.

\section{Case Report}

A 6-year-old boy referred from ENT OPD presented with absence of left external ear since birth, history of decreased hearing, and discharge from left infra-aural sinus since the past 2 years. The discharge was scanty, intermittent, serous, now turned purulent. No history of fever, earache, or rhinitis. No history of maternal infections during pregnancy. The patient had a spontaneous full-term normal vaginal delivery and cried immediately after birth. No history of neonatal intensive care unit stay. On otological examination, left grade III microtia with anotia was found. Atretic left external auditory canal (EAC) was also observed. Puretone audiometry revealed moderate to severe mixed hearing loss in the left ear and normal right ear. published online

July 28, 2021
DOI https://doi.org/

$10.1055 / \mathrm{s}-0041-1734355$ ISSN 0971-3026 (c) 2021. Indian Radiological Association.

This is an open access article published by Thieme under the terms of the Creative Commons Attribution-NonDerivative-NonCommercial-License, permitting copying and reproduction so long as the original work is given appropriate credit. Contents may not be used for commercial purposes, or adapted, remixed, transformed or built upon. (https://creativecommons.org/licenses/by-nc-nd/4.0/). Thieme Medical and Scientific Publishers Private Ltd. A-12, Second Floor, Sector -2, NOIDA -201301, India 
The patient underwent a multiplanar MRI of the brain in our department on a 3.0-Tesla machine using Tl-weighted and T2-weighted fast spin-echo sequences. Additionally, a 3D DRIVE sequence was performed for screening of the inner ear and cranial nerves. It revealed a dysplastic, bulbous, and truncated left-sided vestibule and adjacent part of the lateral semicircular canal (LCC) suggestive of vestibular apparatus malformation ( - Fig. 1A, B). No imaging evidence of CHARGE syndrome was found. The remainder of the left LCC was not appreciated. Cochlea and the rest of the semicircular canals (SCC) appeared unremarkable. Oblique sagittal reconstructions oriented perpendicular to the long axis of the internal auditory canal of the DRIVE sequence was done which revealed nonappreciation of the left facial nerve ( - Fig. 2A, B). The right inner ear was normal. No abnormality was detected in the brain parenchyma.

Limited plain CT sections were taken which revealed normal right ear and multiple anomalies in the left ear. The left pinna was not visualized suggestive of anotia ( - Fig. 3A, B). The left EAC appeared abnormally oriented with atretic cartilaginous portion. Its bony canal was dysmorphic, obliterated with soft tissue, and directed anteroinferiorly with its external opening seen posterior to the left temporomandibular joint (-Fig. 3C, D). The tympanic membrane was not visualized separately. The left middle ear was atretic with dysmorphic malleus and incus and nonvisualization of sta-

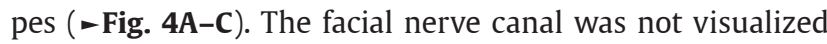
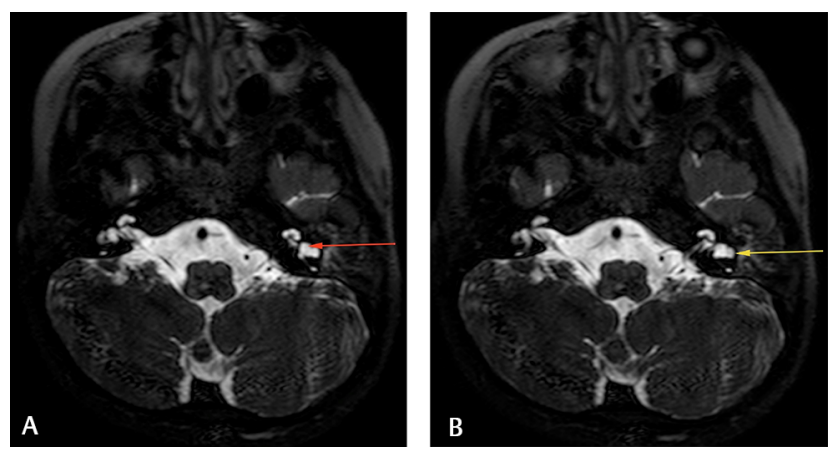

Fig. 1 (A, B) Axial 3T MRI 3D DRIVE showing dysplastic, bulbous, and truncated left-sided vestibule (red arrow) and adjacent part of the LCC (yellow arrow) with nonappreciation of the rest of the LCC suggestive of vestibular system malformation. LCC, lateral semicircular canal; MRI, magnetic resonance imaging.
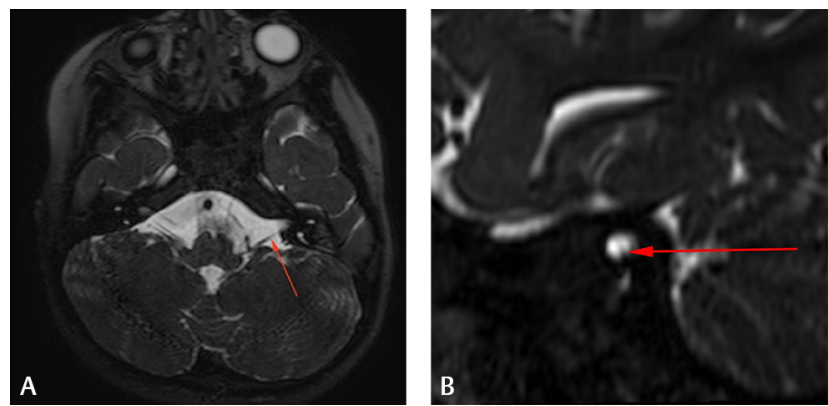

Fig. 2 (A) Axial and (B) oblique sagittal 3D DRIVE showing left vestibulocochlear nerve (red arrow) and nonappreciation of the left facial nerve.
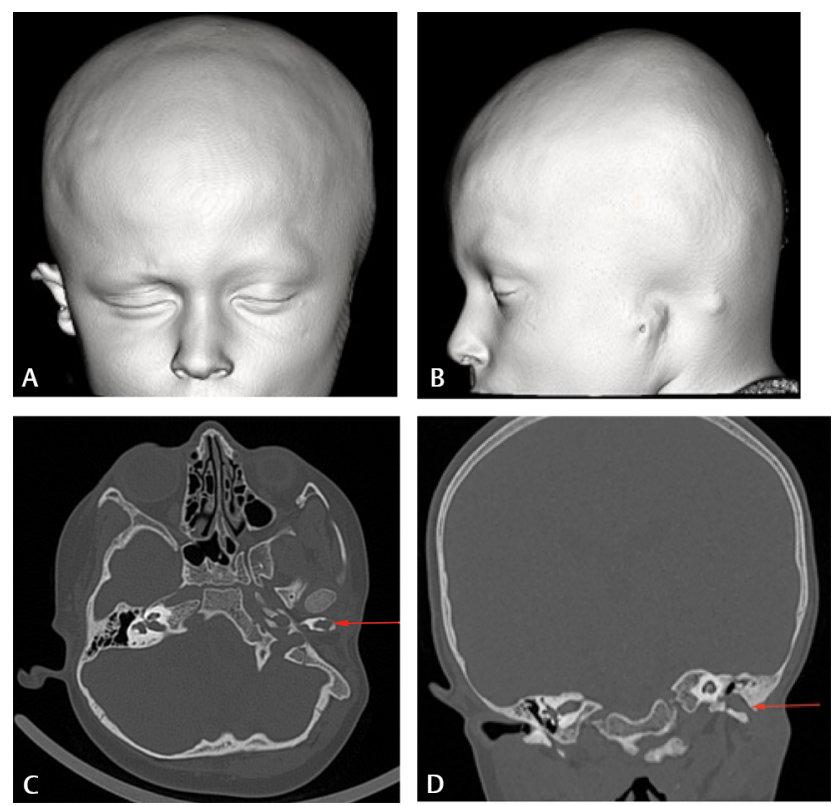

Fig. 3 (A-D) External ear malformation: (A, B) CT (3D reconstruction) of the skull showing left anotia. (C) Axial and (D) coronal CT sections showing nonappreciation of the lateral part of EAC and soft tissue in the medial part of EAC (red arrow). CT, computed tomography; EAC, external auditory canal.
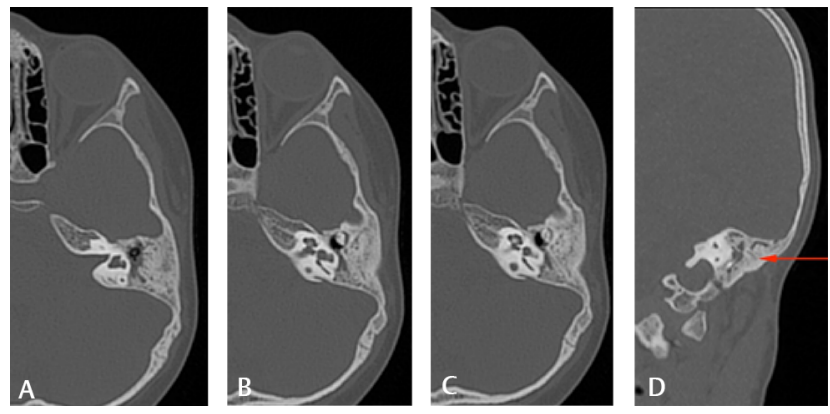

Fig. 4 (A-D) Middle ear malformation. (A) Axial CT showing atretic left middle ear cavity with soft tissue within. (B, C) Axial CT sections showing atretic middle ear cavity with dysmorphic malleus and incus and nonvisualization of stapes. (D) Coronal CT showing sclerosis of left mastoid air cells (red arrow) with soft tissue within. CT, computed tomography.
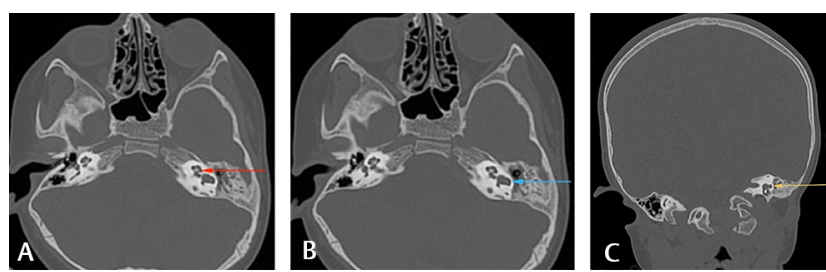

Fig. 5 (A-C) Inner ear malformation. (A) Axial CT showing hyperdense modiolus (red arrow). (B) Axial and (C) coronal CT with a dysplastic, bulbous, and truncated left-sided vestibule (blue arrow) and adjacent part of LCC (yellow arrow) with nonappreciation of the rest of the $\mathrm{LCC}$. CT, computed tomography; LCC, lateral semicircular canal.

in the genu, tympanic, and mastoid segments. The styloid process and stylomandibular foramen were not visualized. Sclerosis/hypopneumatization of left mastoid air cells with 

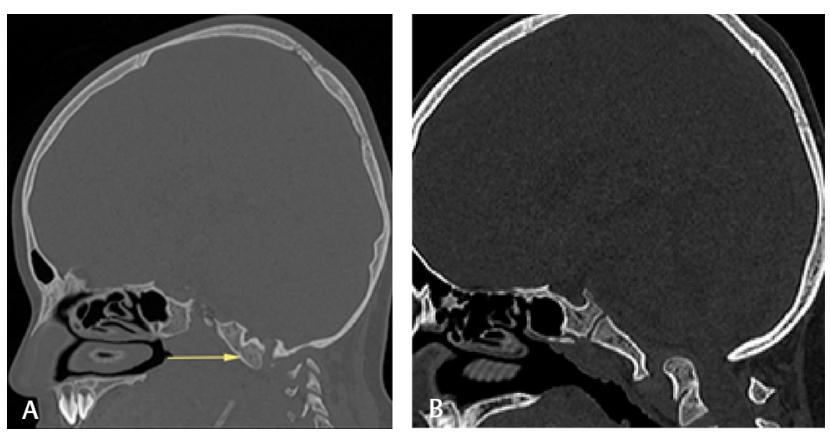

Fig. 6 (A) Sagittal CT reconstruction showing partial occipitalization (yellow arrow) of the right C1 arch. (B) Sagittal CT image showing basilar invagination. CT, computed tomography.

soft tissue opacification of visualized mastoid air cells and middle ear cavity was observed suggestive of otomastoiditis ( - Fig. 4A, D). The modiolus appeared hyperdense with a dysplastic, bulbous, and truncated left-sided vestibule and adjacent part of LCC. The rest of the left LCC was not appreciated


visualized cervical vertebrae in $\mathrm{C} 1$ and $\mathrm{C} 2$ with nonappreciation of right $\mathrm{C} 1$ arch with suspicion of partial occipitalization and basilar invagination ( - Fig. $\mathbf{6 A}, \mathbf{B}$ ). Fusion of $\mathrm{C} 2$ and C3 vertebrae was also seen. These features would most likely represent complex first and second branchial cleft anomalies associated with inner ear malformation.

\section{Discussion}

Our case was unique since usually external ear anomalies are associated with middle anomalies and not with inner ear because of differences in embryogenesis. The incidence of inner ear anomalies reported in patients with EAC atresia varies between 3 and $22 \%{ }^{1,2}$

\section{Embryology}

The ear starts to develop around the third week of gestation from the otic placode which invaginates to form an otic pit, which is then closed to form the otic vesicle from which membranous labyrinth is derived. The bony labyrinth is created from the adjacent mesenchyme. The development of the cochlea is complete by the eighth week.,4 The development of SCCS is completed between the 19th and 22nd weeks. ${ }^{3,4}$

The middle ear development starts in the fourth week. The first pharyngeal pouch together with a contribution from the second pouch forms the tubotympanic recess which gives rise to the middle ear and auditory tube. ${ }^{4}$ The malleus and incus are derived from Meckel's cartilage (cartilage of the first arch), while stapes and styloid process are derived from the cartilage of the second pharyngeal arch. ${ }^{4}$ The facial nerve originates from the otic capsule and second branchial arch.

The external ear is the last part to develop embryologically. Pinna starts developing in the fifth week from six hillocks of His which are the mesenchymal proliferations of the first and second pharyngeal arches. ${ }^{3}$ The first arch gives rise to the tragus and superior portion of the helix, whereas the second arch contributes to antihelix, antitragus, and lobule. ${ }^{4}$
The development of auricle is completed by the 20th week of gestation. ${ }^{3,4}$ The first ectodermal cleft forms the EAC which invaginates medially to meet the endoderm of the middle ear. The mesodermal component gets entrapped leading to the formation of meatal plate.

This plate normally resorbs by the 28th week of gestation causing recanalization of EAC.,3 Failure of recanalization leads to congenital aural atresia.

\section{Anomalies of Ear}

Ear anomalies may be found in isolation or can also be associated with complex syndromes such as Goldenhar's syndrome, branchio-oto-renal syndrome, CHARGE syndrome, Klippel-Feil's syndrome, etc. External and middle ear anomalies are far more likely to coexist due to their closely interrelated development from first and second branchial arches.

Auricular deformities can occur involving single or multiple hillocks with development being arrested at any stage leading to partial or total absence. It may also be associated with preauricular tags or sinuses. Weerda ${ }^{5}$ classified pinna malformations into three grades with increasing severity of the malformations with grade III microtia with anotia being the most severe as seen in our case.

EAC atresia is often seen with anotia and typically involves both cartilaginous and bony parts of EAC. The lumen may be closed by fibrous tissue, cartilage, or bone. Radiological studies are a useful preoperative tool for clinicians in such cases to analyze the tympanic membrane and middle ear structures as they are obscured clinically due to atretic EAC.

Various middle ear anomalies associated with EAC atresia include rudimentary ossicles especially the handle of malleus as the tympanic membrane is usually absent. Abnormal or absent stapes is important as it needs to be replaced with a prosthetic. Hypoplasia of the tympanic cavity is usually seen. Width of more than $3 \mathrm{~mm}$ is usually needed for successful surgical outcome. ${ }^{6}$ Facial nerve injury is the most dangerous complication of reconstructive surgery; hence, its course needs to be delineated preoperatively. The facial nerve tends to have an anterolateral displacement of its course in microtia patients conferring surgical risk. ${ }^{7}$ Both the oval window and round window need to be present for a successful operation. An aberrant course of the internal carotid artery can also be seen which if undetected can lead to hazardous surgical complications. A combined anomaly of the EAC and the middle ear due to their common embryogenesis has been classified according to Altmann ${ }^{5}$ into three degrees with increasing grades of severity.

Knowledge of oval and round windows, the status of ossicles, tympanic cavity size, facial canal, and pneumatization of the mastoid, is needed before recanalization of the atretic EAC is planned. Hence, HRCT of the temporal bone is necessary as a preoperative investigation. A grading system based on preoperative HRCT using eight crucial areas of temporal bone anatomy proposed by Jahrsdoerfer et al is usually used to select the best surgical candidates. The presence of stapes is the most important criterion for atresia surgery. A score of less than 5 is a poor surgical candidate. ${ }^{8}$ 
Most patients with anotia have a well-preserved inner ear. Associated inner ear disorders are usually seen in unilateral aural atresia cases combined with microtia. ${ }^{1}$ The most common inner ear anomaly seen in association is vestibular apparatus malformation particularly that of SCC. ${ }^{1,2}$ Lateral SCC dysplasia is the most common SCC (16.4\%) to be involved. ${ }^{1}$

\section{Conclusion}

Usually, children with malformed pinna have EAC atresia with associated middle ear anomalies. Our case is unique as inner ear anomaly was seen in conjuncture with external ear and middle ear anomalies. Detection of congenital ear malformations is very crucial for the proper growth and development of the child. Imaging findings are indispensable for early diagnosis, accurate classification of the severity of the disease, surgical decision-making, as well as for prognosticating the surgical outcome. Both CT and MRI play a complementary role. Their timely and appropriate usage will thus help in categorization and effective management and in reducing the overall childhood disability burden.

\section{Declaration of Patient Consent}

The authors certify that they have obtained all appropriate patient consent forms. In the form, the patient(s) has/have given his/her/their consent for his/her/their images and other clinical information to be reported in the journal. The patients understand that their names and initials will not be published and due efforts will be made to conceal their identity, but anonymity cannot be guaranteed.

\section{Financial Support and Sponsorship}

Nil.

\section{Conflict of Interest}

None declared.

\section{References}

1 Park E, Lee G, Jung HH, Im GJ. Analysis of inner ear anomalies in unilateral congenital aural atresia combined with microtia. Clin Exp Otorhinolaryngol 2019;12(2):176-180

2 Vrabec JT, Lin JW. Inner ear anomalies in congenital aural atresia. Otol Neurotol 2010;31(9):1421-1426

3 Amarnath C, Sathyan G, Soniya R, Periakaruppan AL, Shankar KS. Evaluation of embryological sequences of ear anomalies and its radiological relevance. Indian J Otol 2016;22:248-257

4 Singh I, Pal GP, The eye and the ear. In: Singh I, ed. Human Embryology. 8th ed. Chennai: Macmillan; 2009 312-318

5 Bartel-Friedrich S, Wulke C. Classification and diagnosis of ear malformations. GMS Curr Top Otorhinolaryngol Head Neck Surg 2007;6:Doc05

6 Gassner EM, Mallouhi A, Jaschke WR. Preoperative evaluation of external auditory canal atresia on high-resolution CT. AJR Am J Roentgenol 2004;182(5):1305-1312

7 Takegoshi H, Kaga K. Difference in facial canal anatomy in terms of severity of microtia and deformity of middle ear in patients with microtia. Laryngoscope 2003;113(4):635-639

8 Jahrsdoerfer RA, Yeakley JW, Aguilar EA, Cole RR, Gray LC. Grading system for the selection of patients with congenital aural atresia. Am J Otol 1992;13(1):6-12 